\title{
hOGG1 Ser326Cys polymorphism and risk of lung cancer by histological type
}

\author{
Toshiki Okasaka ${ }^{1,2}$, Keitaro Matsuo ${ }^{1,3}$, Takeshi Suzuki ${ }^{4}$, Hidemi Ito $^{1}$, Satoyo Hosono ${ }^{1}$, Takakazu Kawase ${ }^{1}$, \\ Miki Watanabe ${ }^{1}$, Yasushi Yatabe ${ }^{5}$, Toyoaki Hida $^{6}$, Tetsuya Mitsudomi ${ }^{7}$, Hideo Tanaka ${ }^{1,3}$, Kohei Yokoi ${ }^{2}$ \\ and Kazuo Tajima ${ }^{1}$
}

\begin{abstract}
Human 8-oxoguanine DNA glycosylase 1 (hOGG1) has a major role in the repair of 8-hydroxyguanine, a major promutagenic DNA lesion. The genetic polymorphism rs1052133, which leads to substitution of the amino acid at codon 326 from Ser to Cys, shows functional differences, namely a decrease in enzyme activity in hOGG1-Cys326. Although several studies have investigated the association between rs1052133 and lung cancer susceptibility, the effect of this locus on lung cancer according to histology remains unclear. We therefore conducted a case-control study with 515 incident lung cancer cases and 1030 age- and sex-matched controls without cancer, and further conducted a meta-analysis. In overall analysis, the homozygous Cys/Cys genotype showed a significant association with lung cancer compared to Ser allele carrier status (odds ratio (OR) $=1.31$, 95\% confidence interval $(\mathrm{Cl})=1.02-1.69)$. By histology-based analysis, the Cys/Cys genotype showed a significantly positive association with small-cell carcinoma $(O R=2.40,95 \% \mathrm{Cl}=1.32-4.49)$ and marginally significant association with adenocarcinoma $(\mathrm{OR}=1.32,95 \% \mathrm{Cl}=0.98-1.77)$. A meta-analysis of previous and our present study revealed that this polymorphism is positively associated with adenocarcinoma, although suggestive associations were also found for squamousand small-cell lung cancers. These results indicate that rs 1052133 contributes to the risk of adenocarcinoma of lung. Journal of Human Genetics (2009) 54, 739-745; doi:10.1038/jhg.2009.108; published online 30 October 2009
\end{abstract}

Keywords: hOGG1; lung cancer; polymorphism

\section{INTRODUCTION}

Cancer is linked to environmental exposure to various carcinogens, of which tobacco smoke is a well-known example. Exposure leads to various types of DNA damage, such as oxidative damage. Genetic variations in DNA repair genes are associated with DNA repair capacity, suggesting a consequent association with cancer risk. ${ }^{1}$

8 -Hydroxyguanine, produced by reactive oxygen species in tobacco smoke, is a major form of DNA damage. ${ }^{2}$ This alteration to the DNA structure causes $\mathrm{G}: \mathrm{C}$ to $\mathrm{T}: \mathrm{A}$ transversions, and may thus be responsible for mutations that lead to carcinogenesis. ${ }^{3}$ Human 8 -oxoguanine DNA glycosylase 1 ( $h O G G 1$ ) has been extensively studied as the main enzyme involved in the repair of 8-oxoG DNA adducts. Although it has a major role in the repair of 8-hydroxyguanine, however, its role in carcinogenesis has not been well elucidated. ${ }^{4}$ Genetic polymorphisms of hOGG1 have been documented, and the polymorphism Ser326Cys (rs1052133) is associated with complementation activity for Escherichia coli mutants that are defective in the repair of 8-hydroxyguanine. Activity in the repair of 8-hydroxyguanine is greater with the hOGG1-Ser326 protein than the hOGG1-Cys326 protein, ${ }^{5}$ and the possible contribution of this locus to the risk of a variety of human cancers has been reported. ${ }^{6}$

A number of studies ${ }^{7-14}$ and systematic approaches ${ }^{15-17}$ have examined the role of the Ser326Cys polymorphism in lung cancer susceptibility. One meta-analysis showed that the overall odds ratio (OR) of homozygotes for the hOGG1-326Cys allele against those for the hOGG1-326Ser allele was 1.24 (95\% confidence interval $(\mathrm{CI})=1.01-1.53)$, suggesting that the locus is involved in susceptibility to overall lung cancer. ${ }^{17}$ In contrast, another meta-analysis reported no significant association. ${ }^{15} \mathrm{~A}$ recent pooled analysis from the International Lung Cancer Consortium involving a substantial number of cases and controls showed a suggestive association for this polymorphism in Caucasians. ${ }^{16}$ One question that remains unanswered is whether the impact of rs1052133 differs according to histological subtype of lung cancer.

Here, we evaluated the role of the hOGG1 Ser326Cys polymorphism in lung cancer susceptibility among a Japanese population in

\footnotetext{
${ }^{1}$ Division of Epidemiology and Prevention, Aichi Cancer Center Research Institute, Nagoya, Japan; ${ }^{2}$ Division of Thoracic Surgery, Nagoya University Graduate School of Medicine, Nagoya, Japan; ${ }^{3}$ Department of Epidemiology, Nagoya University Graduate School of Medicine, Nagoya, Japan; ${ }^{4}$ Department of Medical Oncology and Immunology, Nagoya City University Graduate School of Medical Science, Nagoya, Japan; ${ }^{5}$ Department of Pathology and Molecular Diagnostics, Aichi Cancer Center Central Hospital, Nagoya, Japan; ${ }^{6}$ Department of Thoracic Oncology, Aichi Cancer Center Central Hospital, Nagoya, Japan and ${ }^{7}$ Department of Thoracic Surgery, Aichi Cancer Center Central Hospital, Nagoya, Japan

Correspondence: Dr K Matsuo, Division of Epidemiology and Prevention, Aichi Cancer Center Research Institute, 1-1 Kanokoden, Chikusa-ku, Nagoya 464-8681, Japan. E-mail: kmatsuo@aichi-cc.jp
}

Received 27 July 2009; revised 26 August 2009; accepted 30 September 2009; published online 30 October 2009 
consideration of histology. We also conducted a meta-analysis of the literature to evaluate the impact of this polymorphism by histology.

\section{MATERIALS AND METHODS}

\section{Subjects}

The case subjects were 515 patients who were newly and histologically diagnosed with lung cancer and who had no history of cancer. Controls were randomly selected from among the 2395 cancer-free individuals and matched by age ( \pm 3 years) and sex to cases in a 1:2 case/control ratio. All subjects were recruited within the framework of the Hospital-based Epidemiologic Research Program at Aichi Cancer Center (HERPACC), as described elsewhere, ${ }^{18,19}$ and were exactly the same cohort we reported on in a previous paper. ${ }^{20}$ In brief, information on lifestyle factors was collected using a self-administered questionnaire from all first-visit outpatients at Aichi Cancer Center Central Hospital aged 18-79 who were enrolled in the HERPACC between January 2001 and November 2005. Response was checked by a trained interviewer. Outpatients were also asked to provide blood samples. Each patient was asked about their lifestyle when healthy or before the current symptoms developed. Approximately $95 \%$ of eligible subjects completed the questionnaire and $60 \%$ provide blood samples. The data were loaded into the HERPACC database and routinely linked with the hospitalbased cancer registry system to update the data on cancer incidence. All participants gave written informed consent and the study was approved by institutional ethical committee of Aichi Cancer Center.

\section{Genotyping of hOGG1}

DNA from each sample was extracted from the buffy coat fraction using a BioRobot EZ1 with an EZ1 DNA Blood $350 \mu \mathrm{l}$ kit or QIAamp DNA Blood mini kit (Qiagen KK, Tokyo, Japan). Polymorphisms of hOGG1 Ser326Cys were examined based on TaqMan assays by Applied Biosystems (Foster City, CA, USA). The principle of the TaqMan real-time polymerase chain reaction (PCR) assay system using fluorogenic probes and $5^{\prime}$ nuclease has been described by Livak. ${ }^{21}$ All of the assays were carried out in 96-well PCR plates using a 7500 Fast Real-Time PCR System (Applied Biosystems) coupled with the 7500 Fast System SDS software. Amplification reactions $(5 \mu \mathrm{l})$ were carried out in duplicate with $30 \mathrm{ng}$ of template DNA, $2 \times$ TaqMan Universal Master Mix buffer (Applied Biosystems) and $20 \times$ primer and probe mix (Applied Biosystems). Thermal cycling was initiated with a first denaturation step of $20 \mathrm{~s}$ at $95^{\circ} \mathrm{C}$, and then by 40 cycles of $3 \mathrm{~s}$ at $95^{\circ} \mathrm{C}$ and $30 \mathrm{~s}$ at $62^{\circ} \mathrm{C}$. Genotyping quality was statistically assessed using the Hardy-Weinberg test in our laboratory; when allelic distributions for controls departed from the Hardy-Weinberg frequency, genotyping was assessed using another method.

\section{Consumption of tobacco, alcohol, fruits and vegetables}

Cumulative smoking dose was evaluated as pack-years (PY), the product of the number of packs consumed per day and the number of years of smoking. Smoking habit was entered in the four categories of never, former, and current smokers of $<40$ and $\geqq 40$ PY. Former smokers were defined as those who quit smoking at least 1 year before the survey. Drinking habit was categorized in the three categories of never, former and current drinkers. Former drinkers were defined as those who quit drinking at least 1 year before the survey. Consumption of fruits and vegetables was determined using a semiquantitative food frequency questionnaire (SQFFQ), described in detail elsewhere. ${ }^{22}$ Briefly, the SQFFQ consisted of 47 single food items with frequencies in eight frequency categories. We estimated average daily intake by multiplying the frequency of intake by the serving size of food (in grams). Energy-adjusted intake of fruits and vegetables was calculated by the residual method. ${ }^{23}$ The SQFFQ was validated using a 3-day weighed dietary record as standard, which showed that reproducibility and validity were acceptable. ${ }^{24}$

\section{Statistical analysis}

To assess the strength of associations between hOGG1 polymorphism and risk of lung cancer, we estimated ORs with 95\% CIs, using conditional logistic models adjusted for potential confounders. For stratified analyses exploring interactions, we applied unconditional logistic regression models because matching was not retained after stratification by smoking and drinking habit and carotene intake in conditional models. Fruit and vegetable intake was categorized into three levels by applying thresholds of tertiles among controls. Potential confounders considered in the multivariate analyses were age, sex, smoking habit (never smokers, former smokers, current smokers of less than 40 or 40 or more PY), drinking habit (never, former and current drinkers), total energy intake (as a continuous variable), and dietary fruit and vegetable intake (g per day, tertiles). Missing values for each covariate were treated as dummy variables and were included in the model. Trend for genotype was assessed by application of a score test value for each genotype (0, homozygous for reference allele or combined reference genotypes; 1 , heterozygote or one reference genotype and 2, homozygous nonreference allele or nonreference genotype). Differences in categorized demographic variables between cases and controls were tested by the $\chi^{2}$-test. Mean values for age and total energy intake were compared for cases and controls by Wilcoxon's signed-rank test. Accordance with the Hardy-Weinberg equilibrium was checked for controls using the $\chi^{2}$-test and the exact $P$-value was used to assess any discrepancies between

Table 1 Characteristics of case and control subjects

\begin{tabular}{|c|c|c|c|}
\hline & $\begin{array}{c}\text { Cases } \\
(\mathrm{n}=515) \\
\mathrm{n}(\%)\end{array}$ & $\begin{array}{c}\text { Controls } \\
(\mathrm{n}=1030) \\
\mathrm{n}(\%)\end{array}$ & P-value \\
\hline \multicolumn{4}{|l|}{ Age } \\
\hline$<50$ & $53(10.3)$ & $108(10.5)$ & \\
\hline $50-59$ & $142(27.6)$ & $283(27.5)$ & \\
\hline $60-69$ & $193(37.5)$ & $389(37.8)$ & \\
\hline $70-79$ & $127(24.7)$ & $250(24.3)$ & 1.00 \\
\hline Mean age (range) & $61.9(23-79)$ & $61.8(26-79)$ & 0.87 \\
\hline \multicolumn{4}{|l|}{ Sex } \\
\hline Male & $381(74.0)$ & $762(74.0)$ & \\
\hline Female & $134(26.0)$ & $268(26.0)$ & 1.00 \\
\hline \multicolumn{4}{|l|}{ Smoking (Pack-years) } \\
\hline$<5$ & $136(26.4)$ & $424(41.2)$ & \\
\hline $5-19.9$ & $31(6.0)$ & $118(11.5)$ & \\
\hline $20-39.9$ & $88(17.1)$ & $208(20.2)$ & \\
\hline$>40$ & $258(50.1)$ & $275(26.7)$ & $<0.001$ \\
\hline Unknown & $2(0.4)$ & $5(0.5)$ & \\
\hline \multicolumn{4}{|l|}{ Drinking status } \\
\hline Never & $196(38.1)$ & $378(36.7)$ & \\
\hline Former $^{a}$ & $15(2.9)$ & $56(5.4)$ & \\
\hline Current & $304(59.0)$ & $596(57.9)$ & 0.08 \\
\hline \multicolumn{4}{|c|}{ Fruit/vegetable consumption (g per day) } \\
\hline Tertile $1(<118.4)$ & 199 (38.8) & $342(33.2)$ & \\
\hline Tertile 2 (118.4-211.3) & $140(27.3)$ & $341(33.1)$ & \\
\hline Tertile $3(>211.4)$ & $166(32.4)$ & $341(33.1)$ & 0.03 \\
\hline Unknown & $8(1.6)$ & $6(0.6)$ & \\
\hline Total energy intake (kcal, s.d. $)^{b}$ & $1670(371)$ & $1676(352)$ & 1.00 \\
\hline \multicolumn{4}{|l|}{ Histology } \\
\hline$A D$ & $316(61.4)$ & & \\
\hline $\mathrm{SQ}$ & 91 (17.7) & & \\
\hline SM & $55(10.7)$ & & \\
\hline LA & $40(7.8)$ & & \\
\hline Others & $13(2.5)$ & & \\
\hline
\end{tabular}

Abbreviations: AD, adenocarcinoma; LA, large-cell carcinoma; SM, small-cell carcinoma; $S Q$, squamous-cell carcinoma.

aFormer drinkers were defined as subjects who had quit drinking at least 1 year previously. ${ }^{b}$ Energy-adjusted. 
genotypes and allele frequencies, with a $P$-value of less than 0.05 considered statistically significant. All analyses were performed using STATA version 10.1 (Stata, College Station, TX, USA).

\section{Meta-analysis}

We conducted a meta-analysis of relevant articles reporting associations between the hOGG1 polymorphism and lung cancer in consideration of the histological subtypes adenocarcinoma, squamous-cell carcinoma and small-cell carcinoma. Medline was searched for papers published between January 1995 and March 2009 and indexed with the terms (lung neoplasms AND (hOGG1 OR OGG1)). Inclusion criteria were (1) reporting of ORs or risk ratios calculated by comparing the Ser/Ser to the Cys/Cys or Cys allele carrier according to histological subtype; (2) a cohort, nested case-control, population-based case-control or hospital-based case-control study design and (3) use of cancer-free controls. All potentially relevant papers were independently reviewed by at least two investigators (TO and $\mathrm{KM}$ ) and any disagreements were resolved by consensus. The reference lists of studies identified through the search process were also checked. Among the 65 papers identified through this

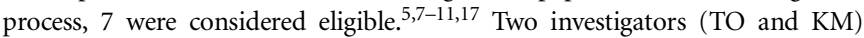
abstracted the data independently. We used OR from a random-effect model as a summary statistic for association. ${ }^{25}$ Heterogeneity among the studies was examined based on the $Q$ and $I^{2}$ statistics. The latter indicates the proportion of variation in summary estimates attributable to heterogeneity. ${ }^{26}$ We determined which model to use to calculate summary OR and its $95 \%$ CI, a random- or fixed-effect model, based on significance in the $Q$ statistics. The meta-analysis was conducted using the 'metan' command ${ }^{27}$ in STATA version 10.1.

\section{RESULTS}

Characteristics of the 515 cases and 1030 controls are shown in Table 1. Age and sex were appropriately matched. Smoking habits differed remarkably between cases and controls, with the proportion of current smokers of 40 PY or more significantly higher in cases. Former drinkers tended to be more common among cases, albeit without statistical significance. Consumption of fruits and vegetables was significantly lower among cases. The distribution of histological type among cases was as follows: adenocarcinoma, $61.4 \%(n=316)$; squamous-cell carcinoma, $17.7 \%(n=91)$; small-cell carcinoma, $10.7 \%$ $(n=55)$; large cell carcinoma, $7.8 \%(n=40)$ and others, $2.5 \%(n=13)$.

Table 2 presents the frequency distribution of hOGG1 genotypes and ORs with 95\% CI for lung cancer cases compared with controls. No significant dissociation from the Hardy-Weinberg equilibrium was observed among controls. In overall analysis, Cys/Cys showed a significantly positive association with lung cancer. The confounderadjusted OR for Cys/Cys relative to Ser/Ser+Ser/Cys was 1.31 (1.021.69, $P=0.036$ ). In histology-based analysis, those with the Cys/Cys genotype were at significantly increased risk of small-cell carcinoma and marginally significantly increased risk of adenocarcinoma, compared to those with the Ser/Cys and Ser/Ser genotypes combined. No significant associations were observed for squamous-cell carcinoma.

Table 3 shows associations between hOGG1 Ser326Cys polymorphism combined with smoking and lung cancer risk. In overall analysis, the effect of cumulative smoking dose was stronger in those with Cys/ Cys. In analyses by histology, a similar trend was observed for adenocarcinoma and small-cell carcinoma but not for squamous-cell carcinoma. This trend was more prominent for small-cell carcinoma. Adjusted ORs for heavy smoking (PY $\geqq 40)$ were 26.3 (5.34-129.6) for the Ser allele carrier and 72.3 (14.6-358.2) for those with the Cys/Cys.

To further examine the impact of $h O G G 1$ Ser326Cys polymorphism according to histology, we conducted a meta-analysis. Table 4 shows a summary of studies that have investigated the association between hOGG1 Ser326Cys polymorphism and lung cancer risk, including the present study. As shown in Figure 1, hOGG1 Ser326Cys polymorphism summary ORs showed a significant association with adenocarcinoma $(\mathrm{OR}=1.44,95 \% \mathrm{CI}=1.18-1.77)$ with no significant heterogeneity. Although squamous-cell carcinoma showed a similarly increased risk $(\mathrm{OR}=1.81,95 \% \mathrm{CI}=1.06-3.07)$, the significant heterogeneity across studies $\left(I^{2}=58.5\right)$ was a limitation. Although without significance and from a limited number of studies, the pooled estimate was 2.05 (0.91-4.63), suggesting an increased risk for small-cell carcinoma.

Table 2 hOGG1 genotype distribution and ORs for lung cancer

\begin{tabular}{|c|c|c|c|c|c|c|}
\hline Genotype & $\begin{array}{c}\text { Cases } \\
\mathrm{n}=515\end{array}$ & $\begin{array}{l}\text { Controls } \\
\mathrm{n}=1030\end{array}$ & OR1 $(95 \% C l)^{a}$ & $\mathrm{P}$-value & OR2 $(95 \% C l)^{\mathrm{b}}$ & P-value \\
\hline \multicolumn{7}{|l|}{ Overall } \\
\hline Ser/Ser & 117 & 250 & 1.00 (reference) & & 1.00 (reference) & \\
\hline Ser/Cys & 257 & 544 & $1.01(0.77-1.32)$ & & $0.96(0.72-1.26)$ & \\
\hline Cys/Cys & 141 & 236 & $1.28(0.94-1.73)$ & 0.054 & $1.27(0.93-1.75)$ & 0.047 \\
\hline Ser/Ser+Ser/Cys & 374 & 794 & 1.00 (reference) & & 1.00 (reference) & \\
\hline Cys/Cys & 141 & 236 & $1.27(1.00-1.62)$ & 0.05 & $1.31(1.02-1.69)$ & 0.036 \\
\hline \multicolumn{7}{|l|}{ Adenocarcinoma } \\
\hline Ser/Ser+Ser/Cys & 227 & 794 & 1.00 (reference) & & 1.00 (reference) & \\
\hline Cys/Cys & 89 & 236 & $1.29(0.97-1.72)$ & 0.085 & $1.32(0.98-1.77)$ & 0.066 \\
\hline \multicolumn{7}{|c|}{ Squamous-cell carcinoma } \\
\hline Ser/Ser+Ser/Cys & 72 & 794 & 1.00 (reference) & & 1.00 (reference) & \\
\hline Cys/Cys & 19 & 236 & $0.99(0.58-1.70)$ & 0.98 & $1.10(0.63-1.94)$ & 0.73 \\
\hline \multicolumn{7}{|l|}{ Small-cell carcinoma } \\
\hline Ser/Ser+Ser/Cys & 34 & 794 & 1.00 (reference) & & 1.00 (reference) & \\
\hline Cys/Cys & 21 & 236 & $2.22(1.26-3.92)$ & 0.006 & $2.40(1.22-4.12)$ & 0.009 \\
\hline
\end{tabular}

Abbreviations: $\mathrm{Cl}$, confidence interval; OR, odds ratio.

aAjusted for age and sex.

Adjusted for age, sex, smoking habit, drinking habit, total energy intake and energy-adjusted fruit/vegetable intake. 
Table 3 Associations between hOGG1 Ser326Cys polymorphisms and smoking by PY on lung cancer risk

\begin{tabular}{|c|c|c|c|c|}
\hline \multirow[b]{2}{*}{ Histology } & \multicolumn{2}{|c|}{$\operatorname{Ser}(+)$} & \multicolumn{2}{|c|}{ Cys/Cys } \\
\hline & Case/Control & OR $(95 \% C l)^{\mathrm{b}}$ & Case/Control & OR $(95 \% C l)^{\mathrm{b}}$ \\
\hline Smoking (pack-years) & & Ser $(+)$ & & Cys/Cys \\
\hline$<5$ & $95 / 317$ & 1.0 (reference) & $41 / 107$ & $1.35(0.88-2.09)$ \\
\hline $5-19.9$ & $22 / 89$ & $1.26(0.72-2.21)$ & $9 / 29$ & $1.29(0.55-3.03)$ \\
\hline \multicolumn{5}{|l|}{ Adenocarcinoma } \\
\hline Smoking & & Ser $(+)$ & & Cys/Cys \\
\hline$<5$ & $89 / 317$ & 1.0 (reference) & $39 / 107$ & $1.36(0.87-2.13)$ \\
\hline 5-19.9 & $15 / 89$ & $0.95(0.50-1.79)$ & $5 / 29$ & $0.70(0.23-2.13)$ \\
\hline 20-39.9 & $39 / 160$ & $1.62(0.98-2.66)$ & $13 / 48$ & $1.75(0.86-3.54)$ \\
\hline \multicolumn{5}{|l|}{ Squamous-cell carcinoma } \\
\hline $5-19.9$ & $3 / 406$ & 1.0 (reference) & $3 / 136$ & $3.22(0.64-16.3)$ \\
\hline 20-39.9 & $13 / 160$ & $6.99(1.93-25.4)$ & $5 / 48$ & $8.99(2.04-39.5)$ \\
\hline$>40$ & $56 / 223$ & $19.5(5.87-64.3)$ & $11 / 52$ & $16.6(4.37-63.0)$ \\
\hline \multicolumn{5}{|l|}{ Small-cell carcinoma } \\
\hline Smoking & & $\operatorname{Ser}(+)$ & & Cys/Cys \\
\hline $5-19.9$ & $2 / 406$ & 1.0 (reference) & $1 / 136$ & $1.50(0.13-16.7)$ \\
\hline 20-39.9 & $8 / 160$ & $12.6(2.39-66.2)$ & $4 / 48$ & $18.8(3.09-114.3)$ \\
\hline$>40$ & $24 / 223$ & $26.3(5.34-129.6)$ & $16 / 52$ & $72.3(14.6-358.2)$ \\
\hline
\end{tabular}

Abbreviations: $\mathrm{Cl}$, confidence intervals; OR, odds ratios.

aFive controls and two cases are excluded from analysis because of smoking information unknown.

${ }^{b}$ ORs were adjusted for age, sex, smoking habit, drinking habit, total energy intake and energy-adjusted fruit/vegetable intake.

Table 4 Summary of published studies examining association between OGG1 polymorphism and lung cancer risk according to histology

\begin{tabular}{|c|c|c|c|c|c|c|c|c|c|c|}
\hline \multirow[b]{3}{*}{ Author } & \multirow[b]{3}{*}{ Year } & \multicolumn{4}{|c|}{ Subjects in each study } & \multirow[b]{3}{*}{ Control } & \multirow[b]{3}{*}{ Ethnicities } & \multirow{2}{*}{\multicolumn{3}{|c|}{ Odds ratio (95\% Cl) for Cys/Cys relative to Ser/Ser }} \\
\hline & & & & ase & & & & & & \\
\hline & & Total & Adeno & Squamous & Small & & & Adeno & Squamous & Small \\
\hline Sugimura et al. ${ }^{7}$ & 1999 & 241 & 1974 & 78 & 118 & 197 & Japanese & $1.34(0.53-3.39)$ & $2.27(0.92-5.60)$ & $0.51(0.09-2.87)$ \\
\hline Wikman et al. ${ }^{8}$ & 2000 & 105 & 50 & 50 & NA & 105 & Caucasian & $1.84(0.41-14.41)$ & $1.76(0.24-13.1)$ & NE \\
\hline Ito et al..$^{9}$ & 2002 & 138 & 138 & 0 & 0 & 241 & Japanese & $0.81(0.44-1.52)$ & NE & NE \\
\hline Le Marchand et al. ${ }^{10}$ & 2002 & 298 & 141 & 66 & 43 & 405 & $\begin{array}{l}\text { Caucasian, } \\
\text { Japanese and } \\
\text { Hawaiian }\end{array}$ & $2.1^{\mathrm{a}}(1.1-3.9)$ & $3.7^{\mathrm{a}}(1.7-8.3)$ & $3.4^{\mathrm{a}}(1.1-10.4)$ \\
\hline Park et al. ${ }^{11}$ & 2004 & 179 & 63 & 56 & 32 & 358 & Caucasian & $4.20(1.10-15.8)$ & $4.8(1.1-21.0)$ & NE \\
\hline Hung et al. ${ }^{17}$ & 2005 & 2188 & 499 & 902 & 0 & 2198 & Caucasian & $1.66(1.04-2.66)$ & $1.02(0.63-1.64)$ & NE \\
\hline Kohno et al. ${ }^{12}$ & 2006 & 1097 & 1097 & 0 & 0 & 394 & Japanese & $1.47(1.02-2.13)$ & NE & NE \\
\hline Our study & 2009 & 515 & 316 & 91 & 55 & 1030 & Japanese & $1.32(0.98-1.77)$ & $1.10(0.63-1.94)$ & $2.40(1.22-4.12)$ \\
\hline
\end{tabular}

Abbreviations: $\mathrm{Cl}$, confidence intervals; $\mathrm{NE}$, not estimated; OR, odd ratios.

aORs are calculated as that of the homozygous Cys/Cys genotype compared to those with the Ser/Ser and Ser/Cys genotype combined.

\section{DISCUSSION}

In this case-control study, we found that the hOGG1 326Cys/Cys genotype, which results in weaker activity, was associated with a significantly increased risk of lung cancer overall. By subtype we found a significant association of the Cys/Cys genotype with small- cell carcinoma and a marginally significant association with adenocarcinoma. Moreover, in our subsequent meta-analysis of epidemiological studies based on histology, we observed that this genotype was associated with an increased risk of adenocarcinoma. Although results for squamous- and small-cell carcinoma were not conclusive, 
a

Adenocarcinoma

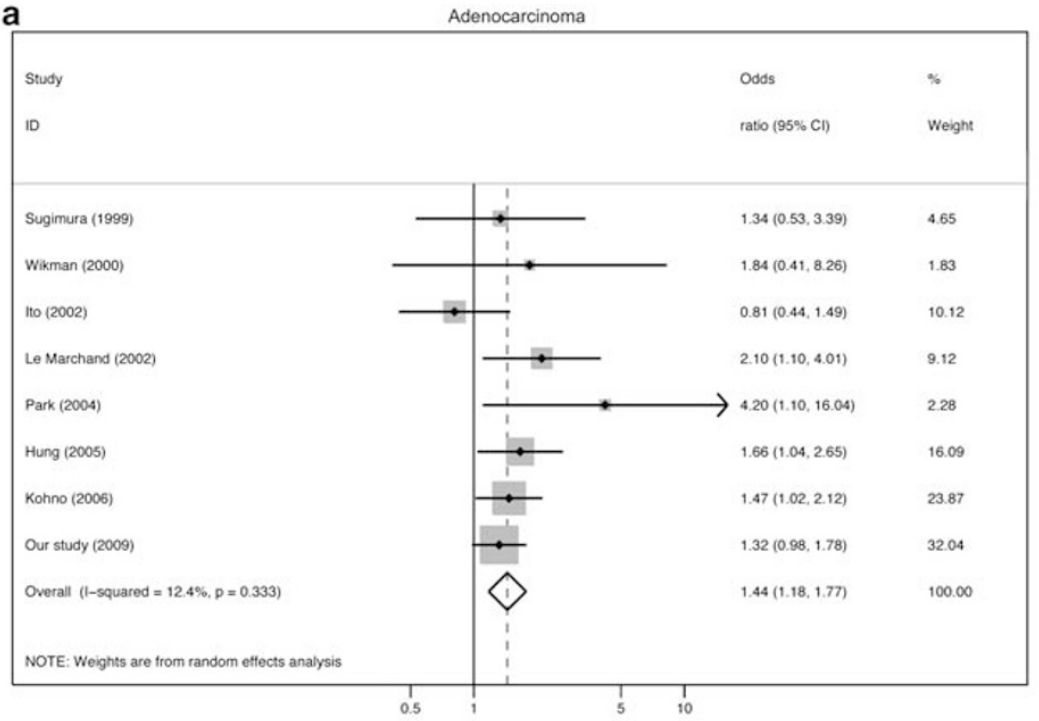

b

Squamous cell carcinoma

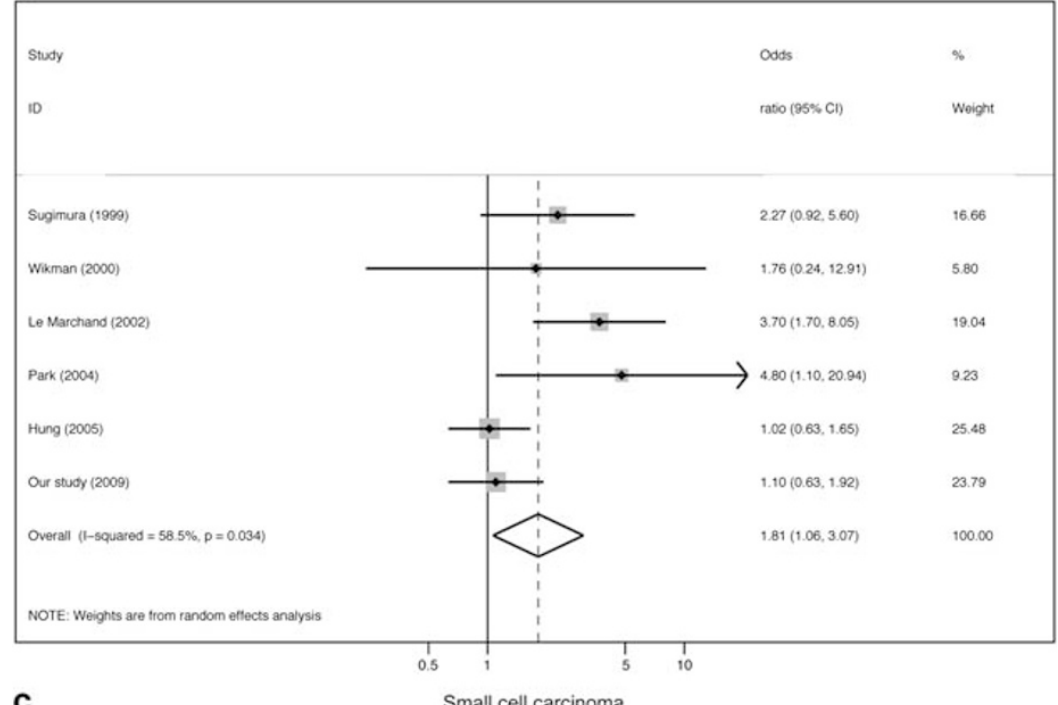

C

Small cell carcinoma

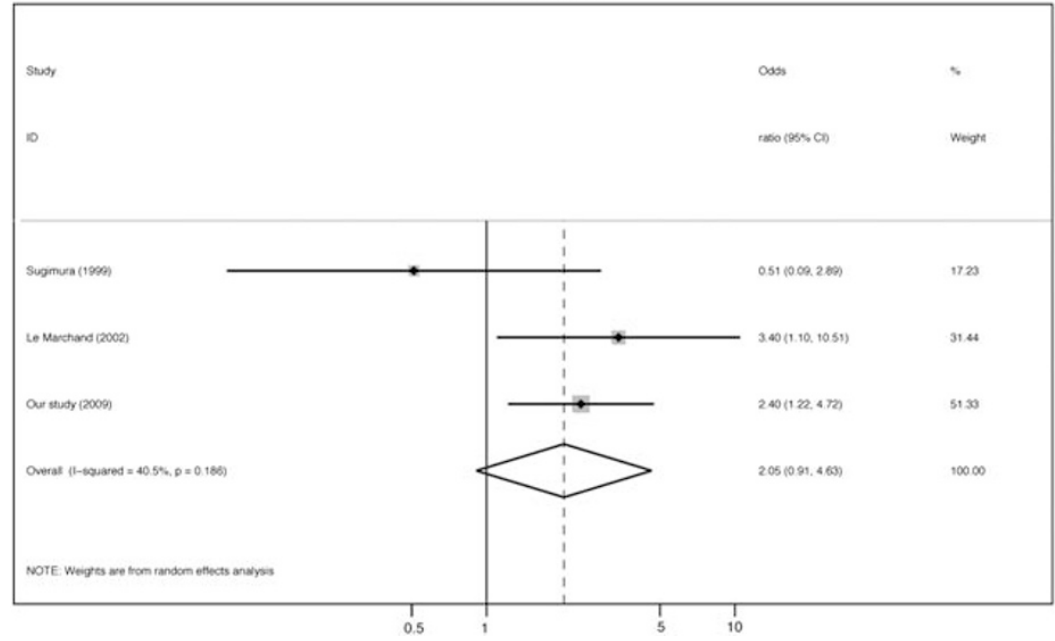

Figure 1 Meta-analysis for human 8-oxoguanine DNA glycosylase 1 (hOGG1) polymorphism according to histological subtype. A meta-analysis was conducted of the studies listed in Table 4. We extracted the ORs and 95\% Cls for the Cys/Cys homozygotes of hOGG1 relative to the Ser/Ser according to histological subtype from each study. We applied a random-effect model. An OR >1.0 indicates a higher risk with the Cys/Cys genotype than with Ser/Ser homozygotes. $R$ indicates the proportion of variation in summary estimates attributable to heterogeneity. All analyses were performed using the 'metan' command in STATA (version 10.1). 
we also identified a potentially increased risk for these types of lung cancer.

Results of a number of studies examining the role of the hOGG1 Ser326Cys polymorphism in lung cancer susceptibility conducted to date have been inconsistent. ${ }^{7-12,17}$ Our case-control study showed a significant association between $h O G G 1$ Ser326Cys polymorphism and lung cancer overall, supporting the potential effect of this polymorphism on lung cancer susceptibility. Because the question of whether the effect of this polymorphism differed by histology remained unanswered, we also conducted a meta-analysis with consideration to histology. To the best of our knowledge, this is the first report to summarize the association between hOGG1 polymorphism and susceptibility by histological type. Results of our meta-analysis indicated that the effect is consistent for adenocarcinoma, but not for squamous- or small-cell carcinoma. This inconsistency might be due to the heterogeneity of populations and distribution of subtypes across studies. The subjects included in the analyses were mainly Japanese and Caucasian. The most common subtype was adenocarcinoma in Japanese but squamous-cell carcinoma in Caucasians. Given that the magnitude of effect of smoking on risk differs by histological subtype ${ }^{28}$ the magnitude of effect of the hOGG1 polymorphism might also differ across subtypes and populations. Even within the same histological subtype, the effect of smoking differs with the presence of certain gene mutations in cancer. ${ }^{29}$ A comprehensive understanding of the hOGG1 polymorphism will thus require further study, with particular focus on squamous- and small-cell carcinomas.

Our case-control study had several potential limitations. One methodological issue was the selection of hospital-based patients without cancer as controls. However, because cases and controls were selected from the same hospital and almost all patients lived in the Tokai area of central Japan, the internal validity of this casecontrol study is likely acceptable. External validity (generalizability of the results) has been confirmed in our previous study. ${ }^{30}$ In addition, to dilute any bias that might have resulted from the inclusion of a specific diagnostic group that is related to the exposure, we did not set eligibility criteria for control diseases. As for allele frequencies in the subjects, given that our frequencies were comparable to those previously reported in public databases such as HapMap JPT, ${ }^{31}$ bias in the distribution of selected polymorphisms was negligible. Second, the self-reported values for lifestyle factors considered as potential confounders may be inaccurate. If present, however, any such misclassification would likely be nondifferential, and would likely underestimate the causal association. The meta-analysis was based on published data, and the potential for publication selection bias could not be ruled out even if heterogeneity across the studies was limited for adenocarcinoma.

In conclusion, we found a positive association between lung cancer and Cys/Cys individuals in a Japanese population. The association was clear for small-cell carcinoma and adenocarcinoma of the lung in this population. Further systematic evaluation revealed that associations with the locus were conclusive for adenocarcinoma. Further studies are needed to clarify the effect of genotype on squamous-cell carcinoma and small-cell carcinoma.

\section{CONFLICT OF INTEREST}

The authors declare no conflict of interest.

\section{ACKNOWLEDGEMENTS}

We thank doctors, nurses, technical staff and hospital administration staff at $\mathrm{ACCH}$ for the daily administration of the Hospital-based Epidemiologic
Research Program at Aichi Cancer Center study. This study was supported by Grants-in-Aid for Scientific Research from the Ministry of Education, Science, Sports, Culture and Technology of Japan, for Cancer Research from the Ministry of Health, Labour and Welfare of Japan, and for the Third Term Comprehensive 10-year Strategy for Cancer Control from the Ministry of Health, Labour and Welfare of Japan.

1 Hoeijmakers, J. H. Genome maintenance mechanisms for preventing cancer. Nature 411, 366-374 (2001).

2 Asami, S., Hirano, T., Yamaguchi, R., Tomioka, Y., Itoh, H. \& Kasai, H. Increase of a type of oxidative DNA damage, 8-hydroxyguanine, and its repair activity in human leukocytes by cigarette smoking. Cancer Res. 56, 2546-2549 (1996).

3 Cheng, K. C., Cahill, D. S., Kasai, H., Nishimura, S. \& Loeb, L. A. 8-Hydroxyguanine, an abundant form of oxidative DNA damage, causes $\mathrm{G}-\mathrm{T}$ and $\mathrm{A}-\mathrm{C}$ substitutions. J Biol. Chem. 267, 166-172 (1992).

4 Boiteux, S. \& Radicella, J. P. The human OGG1 gene: structure, functions, and its implication in the process of carcinogenesis. Arch. Biochem. Biophys. 377, 1-8 (2000).

5 Kohno, T., Shinmura, K., Tosaka, M., Tani, M., Kim, S. R., Sugimura, H. et al. Genetic polymorphisms and alternative splicing of the $h O G G 1$ gene, that is involved in the repair of 8-hydroxyguanine in damaged DNA. Oncogene 16, 3219-3225 (1998).

6 Weiss, J. M., Goode, E. L., Ladiges, W. C. \& Ulrich, C. M. Polymorphic variation in hOGGI and risk of cancer: a review of the functional and epidemiologic literature. Mol. Carcinog. 42, 127-141 (2005).

7 Sugimura, H., Kohno, T., Wakai, K., Nagura, K., Genka, K., Igarashi, H. et al. hOGG1 Ser326Cys polymorphism and lung cancer susceptibility. Cancer Epidemiol. Biomarkers Prev. 8, 669-674 (1999).

8 Wikman, H., Risch, A., Klimek, F., Schmezer, P., Spiegelhalder, B., Dienemann, H. et al. hOGG1 polymorphism and loss of heterozygosity (LOH): significance for lung cancer susceptibility in a Caucasian population. Int. J. Cancer 88, 932-937 (2000).

9 Ito, H., Hamajima, N., Takezaki, T., Matsuo, K., Tajima, K., Hatooka, S. et al. A limited association of OGG1 Ser326Cys polymorphism for adenocarcinoma of the lung. J. Epidemiol. 12, 258-265 (2002).

10 Le Marchand, L., Donlon, T., Lum-Jones, A., Seifried, A. \& Wilkens, L. R. Association of the hOGG1 Ser326Cys polymorphism with lung cancer risk. Cancer Epidemiol. Biomarkers Prev. 11, 409-412 (2002).

11 Park, J., Chen, L., Tockman, M. S., Elahi, A. \& Lazarus, P. The human 8-oxoguanine DNA N-glycosylase 1 (hOGG1) DNA repair enzyme and its association with lung cancer risk. Pharmacogenetics 14, 103-109 (2004).

12 Kohno, T., Kunitoh, H., Toyama, K., Yamamoto, S., Kuchiba, A., Saito, D. et al. Association of the OGG1-Ser326Cys polymorphism with lung adenocarcinoma risk. Cancer Sci. 97, 724-728 (2006).

13 Chang, J. S., Wrensch, M. R., Hansen, H. M., Sison, J. D., Aldrich, M. C., Quesenberry, C. P. Jr. et al. Base excision repair genes and risk of lung cancer among San Francisco Bay Area Latinos and African-Americans. Carcinogenesis 30, 78-87 (2009).

14 Sorensen, M., Raaschou-Nielsen, O., Hansen, R. D., Tjonneland, A., Overvad, K. \& Vogel, U. Interactions between the OGG1 Ser326Cys polymorphism and intake of fruit and vegetables in relation to lung cancer. Free Radic. Res. 40, 885-891 (2006).

15 Li, H., Hao, X., Zhang, W., Wei, Q. \& Chen, K. The hOGG1 Ser326Cys polymorphism and lung cancer risk: a meta-analysis. Cancer Epidemiol. Biomarkers Prev. 17, 1739-1745 (2008).

16 Hung, R. J., Christiani, D. C., Risch, A., Popanda, O., Haugen, A., Zienolddiny, S. et al. International Lung Cancer Consortium: pooled analysis of sequence variants in DNA repair and cell cycle pathways. Cancer Epidemiol. Biomarkers Prev. 17, 3081-3089 (2008).

17 Hung, R. J., Brennan, P., Canzian, F., Szeszenia-Dabrowska, N., Zaridze, D., Lissowska, J. et al. Large-scale investigation of base excision repair genetic polymorphisms and lung cancer risk in a multicenter study. J. Natl. Cancer Inst. 97, 567-576 (2005).

18 Tajima, K., Hirose, K., Inoue, M., Takezaki, T., Hamajima, N. \& Kuroishi, T. A model of practical cancer prevention for out-patients visiting a hospital: the Hospital-based Epidemiologic Research Program at Aichi Cancer Center (HERPACC). Asian Pac. J. Cancer Prev. 1, 35-47 (2000).

19 Hamajima, N., Matsuo, K., Saito, T., Hirose, K., Inoue, M., Takezaki, T. et al. Gene-environment interactions and polymorphism studies of cancer risk in the Hospital-based Epidemiologic Research Program at Aichi Cancer Center II (HERPACC-II). Asian Pac. J. Cancer Prev. 2, 99-107 (2001).

20 Suzuki, T., Matsuo, K., Hiraki, A., Saito, T., Sato, S., Yatabe, Y. et al. Impact of onecarbon metabolism-related gene polymorphisms on risk of lung cancer in Japan: a case control study. Carcinogenesis 28, 1718-1725 (2007).

21 Livak, K. J. Allelic discrimination using fluorogenic probes and the 5' nuclease assay. Genet. Anal. 14, 143-149 (1999).

22 Tokudome, S., Ikeda, M., Tokudome, Y., Imaeda, N., Kitagawa, I. \& Fujiwara, N. Development of data-based semi-quantitative food frequency questionnaire for dietary studies in middle-aged Japanese. Jpn. J. Clin. Oncol. 28, 679-687 (1998).

23 Ma, J., Stampfer, M. J., Giovannucci, E., Artigas, C., Hunter, D. J., Fuchs, C. et al. Methylenetetrahydrofolate reductase polymorphism, dietary interactions, and risk of colorectal cancer. Cancer Res. 57, 1098-1102 (1997). 
24 Tokudome, Y., Goto, C., Imaeda, N., Hasegawa, T., Kato, R., Hirose, K. et al. Relative validity of a short food frequency questionnaire for assessing nutrient intake versus three-day weighed diet records in middle-aged Japanese. J. Epidemiol. 15, 135-145 (2005).

25 DerSimonian, R. \& Laird, N. Meta-analysis in clinical trials. Control. Clin. Trials 7, 177-188 (1986).

26 Higgins, J. P., Thompson, S. G., Deeks, J. J. \& Altman, D. G. Measuring inconsistency in meta-analyses. BMJ 327, 557-560 (2003).

27 Harris, R. J., Bradburn, M. J., Deeks, J., Harbord, R., Altman, D. G. \& Sterne, J. A. C. Metan: fixed- and random-effects meta-analysis. Stata J. 8, 3-28 (2008).
28 Lubin, J. H. \& Caporaso, N. E. Cigarette smoking and lung cancer: modeling total exposure and intensity. Cancer Epidemiol. Biomarkers Prev. 15, 517-523 (2006).

29 Matsuo, K., Ito, H., Yatabe, Y., Hiraki, A., Hirose, K., Wakai, K. et al. Risk factors differ for non-small-cell lung cancers with and without EGFR mutation: assessment of smoking and sex by a case-control study in Japanese. Cancer Sci. 98, 96-101 (2007).

30 Inoue, M., Tajima, K., Hirose, K., Hamajima, N., Takezaki, T., Kuroishi, T. et al. Epidemiological features of first-visit outpatients in Japan: comparison with general population and variation by sex, age, and season. J. Clin. Epidemiol. 50, 69-77 (1997).

31 International HapMap Consortium. A haplotype map of the human genome. Nature 437, 1299-1320 (2005). 